\title{
Online Learning Using The Zenius App At Madrasah Ibtidaiyah Negeri 8 (MIN) Central Aceh
}

\author{
Suwarno ${ }^{1}$, Firmansyah ${ }^{2}$, Andika Hariyanto Surbakti ${ }^{3}$ Indra $^{4}$, Muchamad Suradji ${ }^{5}$ \\ \{fauzansuwarno@gmail.com, fmanb88@gmail.com,Andikahariyantosurbakti1983@gmail.com, \\ indragayo1@gmail.com, msuradji@unisda.ac.id\} \\ 1,2,3,4 IAIN Takengon, ${ }^{5}$ Universitas Islam Darul 'Ulum Lamongan
}

\begin{abstract}
Zenius is an application developed for distance learning. This application has several advantages, including the availability of subject matter and learning videos. This study aimed to determine the effectiveness of using the Zenius application in online learning at MIN 8 Central Aceh. The research method used in this study is a level 2 development research method, according to Sugiono, namely researching to test existing products. Data collection is done using observation, interviews, and tests. Observations and interviews were conducted at the literature study stage as the first step in level 2 development research. At the same time, the tests used were pretest and posttest - data analysis techniques using quantitative analysis. The results showed that from the limited Trial, the pretest and posttest scores increased with a high significance of $16.67 \%, 0 \%$, and low, $83.33 \%$. Then it can be said to be effective. In the usage trial, it is known that there is an increase in the pretest and posttest scores with a high significance level of $30 \%$, moderate $0 \%$, and low $70 \%$; it is stated that the use of the Zenius application in learning is effective. The broad class trial found an increase in the pretest and posttest scores with a high significance of $35.53 \%$, moderate $0 \%$, and low $64,47 \%$. It is stated that the application used in online learning is practical.
\end{abstract}

Keywords: Learning, Online, Application, Zenius

\section{Introduction}

Corona virus infection is a disease caused by a coronavirus and causes the main symptoms of respiratory problems. This disease is in the spotlight because of its emergence at the end of 2019 for the first time in Wuhan, China. For the first time, the location of its appearance has made the COVID-19 Covid-19. Symptoms of Covid-19 range from the flu to severe diseases such as Middle East Respiratory Syndrome or what is known as MERS-CoV and Severe Acute Respiratory Syndrome or SARS-CoV.[1] A new type of coronavirus causes Covid-19. This virus is a new type of virus, so this disease was unknown until the Covid-19 outbreak in Wuhan, China, in December 2019. Cases of Covid-19 disease appeared and infected humans for the first time in Wuhan, China. Covid-19 can be spread easily through coughing or breath released by a person with Covid-19. 
Splashes of coughs and breaths by Covid-19 sufferers that fall onto the surface of an object can transmit the disease through the thing.[1]. If a person touches an object or inhales the splash then touches his nose, eyes, or mouth, he can contract Covid-19.

The world health organization, the World Health Organization (WHO), urges people to maintain a distance of more than 1 meter from other people to minimize the transmission of Covid19. The information of Covid-19 is so fast that the World Health Organization (WHO) designated this coronavirus or Covid-19 as a pandemic on March 11, 2020. This global epidemic status or pandemic indicates that the spread of Covid-19 is taking place very quickly. Almost no country in the world can avoid the coronavirus (Mona, 2020), so that governments in various countries have implemented lockdowns or quarantines in the form of separating someone who has been exposed to COVID-19, including Indonesia, as stated by Suwarno, Indonesia, as one of the countries exposed to COVID-19 also implemented a similar policy. Since the first case of COVID-19 was confirmed on March 2, 2020, in Depok, West Java, the number of positive cases continues to grow quite quickly.[2]

The Indonesian government has implemented the Large-Scale Social Restrictions (PSBB) rules to control the spread of Covid-19. This is done hoping that the virus does not spread more widely and healing efforts can run optimally. In this social restriction effort, the Indonesian government has limited activities outside the home, such as educational activities carried out online (online) through online learning since March 2020, referring to the circular letter of the Minister of Education and Culture Number 4 of 2020. Online learning is carried out by utilizing internet technology with The goal is to reduce crowds to prevent the spread of the Covid-19 virus. Online learning is carried out with a distance learning system, namely between teachers and students, not in one room or one place.

Online learning is a new learning model in Indonesia. So many problems occur in the field, as stated by Zain that the limited material received by students makes learning not optimal.[3]. Other problems Students are not able to understand the content of the material that has been presented through online media by the teacher; the internet network is sometimes disrupted, the lack of use of online learning media so that the teacher cannot convey some subject matter that requires specific learning tools and media the maximum.[4] In terms of readiness, as stated by Jauhari, the problem that arises is that the preparation of teachers in making learning tools is a little more complicated; in online learning, teachers are required to master Information Technology (IT) considering that learning is not done face-to-face.[5]. Furthermore, Jauhari revealed that teachers are tired of doing online learning considering a large number of students, different schedules are made so that many teachers are not ready to do online learning, especially to prepare online learning media based on information technology using android applications.[5], In addition, the factor of unpreparedness of educators is because they are not familiar with online learning and have not been able to use existing platforms or applications used in online learning. This is a particular problem for those who do not understand using IT. (Rudi Haryadi, 2021)

From the description above, it can be understood that there are still teachers who are not ready to do online learning, have difficulty making learning tools, preparing materials, and so on, which is happening in many areas. The same thing happened in MIN 8 Central Aceh. Departing from these problems, the author is interested in researching the use of the Zenius application in online learning; Zenius is a free platform that can be accessed by all teachers in Indonesia, launched for the first time to coincide with National Education Day 2020. Zenius is a free Learning 
Management System created by teachers for teachers. Zenius claims that this platform can be used by teachers throughout Indonesia for free, by teachers of all subjects, at all levels of education to facilitate the management of learning activities. Zenius Application Development is a form of Zenus' mission to improve Indonesian educational competence globally. This edutech startup also intends to create a more thoughtful, brighter, and more fun Indonesia. Because the application has available learning materials, the teacher can start classes to overcome the material's difficulties. This study aimed to determine the effectiveness of using the Zenius application in online learning at MIN 8 Bebesen Central Aceh.

\section{Research Methods}

This research uses the level 2 development research method; according to Sugiono, level 2 development research is development research that aims to test existing products (Sugiyono, 2017), here the position of the researcher is only to try, did not research to test the effectiveness of current products. . Level 2 research and development steps include several stages. First, using a particular product, here the product in question is the Zenius application for online learning. Second, literature studies; in this step, the researchers conducted field observations and Interviews related to online learning at MIN 8 Central Aceh. The third Trial, trials were conducted in small groups, use trials, and broad trials. Small group trials were carried out on students in small groups (sample selected at random). The practice of use by carrying out learning using the Zenius application classically in one class of students. Extensive trials by carrying out education using the Zenius application classically in three categories. Fourth, make a test report. analyze the test results, and make conclusions. (Rudi Haryadi, 2021). The data collection technique uses the tets approach to determine the effectiveness of using the Zenius application in online learning. The data analysis used is quantitative analysis to evaluate the effectiveness of using the Zenius application.

\section{Results and Discussion}

\section{Results of the literature study}

At the literature study stage in this level 2 development research, the researchers made observations at the research location, namely at MIN 8 Central Aceh; MIN 8 Central Aceh is one of the basic level Madrasah under the auspices of the Ministry of Religion of Central Aceh which actively implements online learning through the manager application. Group, the whatshap group, google classroom, and zoom cloud meeting, since March 26, 2020. However, according to the Head of MIN 8 Central Aceh, Irfan, S.Pd.I, although Madrasah is actively implementing online learning, it turns out there are several obstacles, including the lack of teacher readiness to prepare online learning tools. So that it impacts decreasing the absorption of subject matter to students, all students 
can take part in the online learning system. Responding to this, the researcher then offers to use the application that Zenius has developed in online learning.

Zenius provides online learning channels for elementary to high school students. Zenius App has three advantages, which are complete, practical, and affordable. Exclusive means that subject matter is available from grade 1 Elementary School to 12 Senior High School according to the education unit level curriculum, 2013 curriculum, and 2013 revised curriculum. There are 80,000 videos and hundreds of thousands of questions from all classes and lessons.

\section{Limited trial results}

In a limited trial, online learning was carried out using the Zenius application for six students in class 3; the tiny trial stage was carried out by conducting an initial test or pretest before conducting online learning, then online learning was carried out using the Zenius application for one week, after learning using the application genius then carried out a final test, or posttest. The results of the pretest and posttest are presented in the following table:

Table 1. Limited pretest and posttest scores

\begin{tabular}{|c|c|r|c|}
\hline \multirow{2}{*}{ No } & \multirow{2}{*}{ Student } & \multicolumn{2}{|c|}{ Value } \\
\cline { 3 - 4 } & & pretest & posttest \\
\hline 1 & A & 54 & 77 \\
\hline 2 & B & 62 & 87 \\
\hline 3 & C & 71 & 94 \\
\hline 4 & D & 51 & 81 \\
\hline 5 & E & 53 & 82 \\
\hline 6 & F & 47 & 76 \\
\hline
\end{tabular}

\section{Descriptive Statistical Analysis}




\section{Descriptives}

\begin{tabular}{|c|c|c|c|c|}
\hline & & & Statistic & Std. Error \\
\hline \multirow[t]{13}{*}{ Nilai Pretest } & Mean & & 56.3333 & 3.55590 \\
\hline & \multirow{2}{*}{$\begin{array}{l}95 \% \text { Confidence Interval } \\
\text { for Mean }\end{array}$} & Lower Bound & 47.1926 & \\
\hline & & Upper Bound & 65.4741 & \\
\hline & \multicolumn{2}{|l|}{$5 \%$ Trimmed Mean } & 56.0370 & \\
\hline & \multicolumn{2}{|l|}{ Median } & 53.5000 & \\
\hline & \multicolumn{2}{|l|}{ Variance } & 75.867 & \\
\hline & \multicolumn{2}{|l|}{ Std. Deviation } & 8.71015 & \\
\hline & \multicolumn{2}{|l|}{ Minimum } & 47.00 & \\
\hline & \multicolumn{2}{|l|}{ Maximum } & 71.00 & \\
\hline & \multicolumn{2}{|l|}{ Range } & 24.00 & \\
\hline & \multicolumn{2}{|l|}{ Interquartile Range } & 14.25 & \\
\hline & \multicolumn{2}{|l|}{ Skewness } & 1.054 & .845 \\
\hline & \multicolumn{2}{|l|}{ Kurtosis } & .543 & 1.741 \\
\hline \multirow[t]{13}{*}{ Nilai Posttest } & \multicolumn{2}{|l|}{ Mean } & 82.8333 & 2.74975 \\
\hline & \multirow{2}{*}{$\begin{array}{l}95 \% \text { Confidence Interval } \\
\text { for Mean }\end{array}$} & Lower Bound & 75.7649 & \\
\hline & & Upper Bound & 89.9018 & \\
\hline & \multicolumn{2}{|l|}{$5 \%$ Trimmed Mean } & 82.5926 & \\
\hline & \multicolumn{2}{|l|}{ Median } & 81.5000 & \\
\hline & \multicolumn{2}{|l|}{ Variance } & 45.367 & \\
\hline & \multicolumn{2}{|l|}{ Std. Deviation } & 6.73548 & \\
\hline & \multicolumn{2}{|l|}{ Minimum } & 76.00 & \\
\hline & \multicolumn{2}{|l|}{ Maximum } & 94.00 & \\
\hline & \multicolumn{2}{|l|}{ Range } & 18.00 & \\
\hline & \multicolumn{2}{|l|}{ Interquartile Range } & 12.00 & \\
\hline & \multicolumn{2}{|l|}{ Skewness } & .923 & .845 \\
\hline & \multicolumn{2}{|l|}{ Kurtosis } & .280 & 1.741 \\
\hline
\end{tabular}

\begin{tabular}{|l|l|l|l|}
\hline $\begin{array}{l}\text { Category } \\
\text { improvement }\end{array}$ & Category N-Gain & $\begin{array}{c}\text { Many } \\
\text { students }\end{array}$ & Percentage \\
\hline
\end{tabular}




\begin{tabular}{|c|c|c|c|}
\hline High & N-Gain $>0,7$ & 1 & $16.67 \%$ \\
\hline Low & $0,3=<$ N-Gain $=<0,7$ & 5 & $83.33 \%$ \\
\hline Medium & N-Gain $<0,3$ & 0 & $0.00 \%$ \\
\hline & & & $100.00 \%$ \\
\hline
\end{tabular}

\section{Data Normality Prerequisite Test}

From the pretest and posttest data, the normality of the data will be tested. Normality test or prerequisite test is used to test whether the variables are normally distributed or not to perform the t-test. If the data is not normally distributed, then the researcher must modify it first, but if it is usually distributed, then immediately perform the core test or t-test. The following is the calculation of the data normality test using SPSS.

\section{Tests of Normality}

\begin{tabular}{|l|r|r|r|r|r|r|}
\hline & \multicolumn{3}{|c|}{ Kolmogorov-Smirnov $^{\text {a }}$} & \multicolumn{3}{c|}{ Shapiro-Wilk } \\
\cline { 2 - 7 } & Statistic & \multicolumn{1}{c|}{$\mathrm{df}$} & \multicolumn{1}{c|}{ Sig. } & Statistic & \multicolumn{1}{c|}{ df } & \multicolumn{1}{c|}{ Sig. } \\
\hline Nilai Pretest & .272 & 6 & .186 & .913 & 6 & .455 \\
Nilai Posttest & .216 & 6 & $.200^{*}$ & .923 & 6 & .527 \\
\hline
\end{tabular}

*. This is a lower bound of the true significance.

a. Lilliefors Significance Correction

Value of Sig. (P-Value) in both data groups $>0.05$, it can be concluded that the scores on both tests (pretest and posttest) are typically distributed.

\section{T-test (paired samples t-test)}

Paired Samples Correlations
\begin{tabular}{|ll|r|r|r|}
\hline & \multicolumn{1}{|c|}{ N } & Correlation & \multicolumn{1}{c|}{ Sig. } \\
\hline Pair 1 & $\begin{array}{l}\text { Nilai Pretest \& Nilai } \\
\text { Posttest }\end{array}$ & 6 & .945 & .004 \\
\hline
\end{tabular}

Value of Sig. $($ P-Value $)=0.004<0.05$, it can be concluded that the two groups of data are correlated

\begin{tabular}{|c|c|c|c|c|c|c|c|c|c|}
\hline & & \multicolumn{5}{|c|}{ Paired Differences } & \multirow[b]{3}{*}{ t } & \multirow[b]{3}{*}{ df } & \multirow[b]{3}{*}{ Sig. (2-tailed) } \\
\hline & & \multirow[b]{2}{*}{ Mean } & \multirow[b]{2}{*}{ Std. Deviation } & \multirow{2}{*}{$\begin{array}{l}\text { Std. Error } \\
\text { Mean }\end{array}$} & \multicolumn{2}{|c|}{$\begin{array}{l}95 \% \text { Confidence Interval of the } \\
\text { Difference }\end{array}$} & & & \\
\hline & & & & & Lower & Upper & & & \\
\hline Pair 1 & $\begin{array}{l}\text { Nilai Pretest - Nilai } \\
\text { Posttest }\end{array}$ & -26.50000 & 3.20936 & 1.31022 & -29.86802 & -23.13198 & -20.226 & 5 & .000 \\
\hline
\end{tabular}

Sig. (2-tailed) / p-value $<0.05$, it can be concluded that there is a significant difference in value between before and after treatment in a limited trial 


\section{Trial use}

In the trial use, online learning was carried out using the Zenius application to 30 class 4 students; the Trial was carried out by conducting a pretest before doing online education, then online learning using the Zenius application was carried out for one week, after learning using the Zenius application was then carried out a final test. Or posttest. The results of the pretest and posttest are presented in the following table:

Table 2. The value of the pretest and posttest of the usage trial

\begin{tabular}{|c|c|c|c|}
\hline NO & STUDENTS NAME & PRETEST & POSTTEST \\
\hline 1 & Achmad Abi Thofani & 50.00 & 78 \\
\hline 2 & Achmad Ragil Surya Firnandha & 44.00 & 79 \\
\hline 3 & Agung Wicaksono Abdullah Putra & 42.00 & 78 \\
\hline 4 & Akhmad Mukhyiddin Arga Pratama & 42.00 & 81 \\
\hline 5 & Arif Kuswardana & 34.00 & 76 \\
\hline 6 & Aulia Dwi Rahmawati & 34.00 & 76 \\
\hline 7 & Aura Dewi Budi Lestari & 36.00 & 84 \\
\hline 8 & Chayla Ezra May Anjani & 38.00 & 78 \\
\hline 9 & Daniel Oktavianto & 34.00 & 78 \\
\hline 10 & Diwantaka Kusuma Putra & 28.00 & 78 \\
\hline 11 & Farel Agustian Crisjianto & 46.00 & 78 \\
\hline 12 & Forza Hakim Saputra & 42.00 & 78 \\
\hline 13 & Heni Kumala Pertiwi & 34.00 & 82 \\
\hline 14 & Imroatul Azizah & 44.00 & 84 \\
\hline 15 & Titania Kusherawati & 52.00 & 86 \\
\hline 16 & Jeni Veronica Anggun Zahraini & 40.00 & 84 \\
\hline 17 & Madani Firmansyah & 38.00 & 85 \\
\hline 18 & Muhamad Aldian Syaputra & 38.00 & 76 \\
\hline 19 & Muhammad Alvin & 58.00 & 84 \\
\hline 20 & Muhammad Bagas Setyawan & 42.00 & 85 \\
\hline 21 & Muhammad Iqbal Ikhwanto & 34.00 & 85 \\
\hline 22 & Mukhammad Junaidi Irgi Dwitama & 42.00 & 81 \\
\hline 23 & Risk Rismawati & 36.00 & 87 \\
\hline 24 & Rehan Bagus Saputra & 46.00 & 75 \\
\hline
\end{tabular}




\begin{tabular}{|l|l|c|c|}
25 & Rehani Nur Ramadani & 40.00 & 76 \\
\hline 26 & Rendyansah Yoga Adi Pratama & 42.00 & 76 \\
\hline 27 & Salsabila Hilda Agustin & 56.00 & 76 \\
\hline 28 & Saverio Noviansyah & 48.00 & 76 \\
\hline 29 & Umrotus Sholikhah & 44.00 & 79 \\
\hline 30 & Vanesa Adelia Putri & 58.00 & 82 \\
\hline
\end{tabular}

\section{Descriptive Statistical Analysis}

\section{Descriptives}

\begin{tabular}{|c|c|c|c|c|}
\hline & & & Statistic & Std. Error \\
\hline \multirow[t]{13}{*}{ Nilai Pretest } & Mean & & 42.0667 & 1.35437 \\
\hline & \multirow{2}{*}{$\begin{array}{l}95 \% \text { Confidence Interval } \\
\text { for Mean }\end{array}$} & Lower Bound & 39.2967 & \\
\hline & & Upper Bound & 44.8367 & \\
\hline & $5 \%$ Trimmed Mean & & 41.8519 & \\
\hline & Median & & 42.0000 & \\
\hline & Variance & & 55.030 & \\
\hline & Std. Deviation & & 7.41821 & \\
\hline & Minimum & & 28.00 & \\
\hline & Maximum & & 58.00 & \\
\hline & Range & & 30.00 & \\
\hline & Interquartile Range & & 10.00 & \\
\hline & Skewness & & .593 & .427 \\
\hline & Kurtosis & & .108 & .833 \\
\hline \multirow[t]{13}{*}{ Nilai Posttest } & Mean & & 80.0333 & .67888 \\
\hline & \multirow{2}{*}{$\begin{array}{l}95 \% \text { Confidence Interval } \\
\text { for Mean }\end{array}$} & Lower Bound & 78.6449 & \\
\hline & & Upper Bound & 81.4218 & \\
\hline & $5 \%$ Trimmed Mean & & 79.9259 & \\
\hline & Median & & 78.5000 & \\
\hline & Variance & & 13.826 & \\
\hline & Std. Deviation & & 3.71839 & \\
\hline & Minimum & & 75.00 & \\
\hline & Maximum & & 87.00 & \\
\hline & Range & & 12.00 & \\
\hline & Interquartile Range & & 8.00 & \\
\hline & Skewness & & .386 & .427 \\
\hline & Kurtosis & & -1.321 & .833 \\
\hline
\end{tabular}




\begin{tabular}{|c|c|c|c|}
\hline Category improvement & Category N-Gain & $\begin{array}{c}\text { Many } \\
\text { students }\end{array}$ & Percentage \\
\hline High & N-Gain $>0,7$ & 9 & $30.00 \%$ \\
\hline Low & $0,3=<$ N-Gain $=<0,7$ & 21 & $70.00 \%$ \\
\hline Medium & N-Gain $<0,3$ & 0 & $0.00 \%$ \\
\hline & & & $100.00 \%$ \\
\hline
\end{tabular}

\section{Data Normality Prerequisite Test}

From the pretest and posttest data, the normality of the data will be tested. Normality test or prerequisite test is used to test whether the variables are normally distributed or not to perform the t-test. If the data is not normally distributed, then the researcher must modify it first, but if it is usually distributed, then immediately perform the core test or t-test. The following is the calculation of the data normality test using SPSS.

Tests of Normality

\begin{tabular}{|l|r|r|r|r|r|r|}
\hline & \multicolumn{3}{|c|}{ Kolmogorov-Smirnov $^{\mathrm{a}}$} & \multicolumn{3}{c|}{ Shapiro-Wilk } \\
\cline { 2 - 7 } & Statistic & \multicolumn{1}{c|}{ df } & \multicolumn{1}{c|}{ Sig. } & \multicolumn{1}{c|}{ Statistic } & \multicolumn{1}{c|}{ df } & \multicolumn{1}{c|}{ Sig. } \\
\hline Nilai Pretest & .137 & 30 & .158 & .946 & 30 & .130 \\
Nilai Posttest & .208 & 30 & .002 & .892 & 30 & .005 \\
\hline
\end{tabular}

a. Lilliefors Significance Correction

Value of Sig. (P-Value) at the posttest value $=0.002<0.05$, it can be concluded that the posttest value is not normally distributed. So the difference test does not use the t-test, but the different test with nonparametric statistics.

\section{T-test (paired samples t-test)}

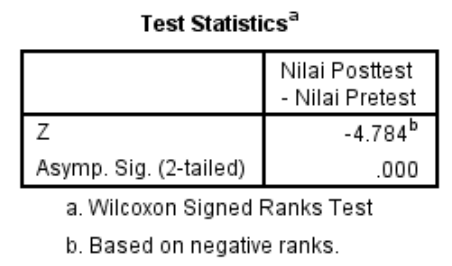

asymp. Sig. (2-tailed)/p-value $<0.05$, it can be concluded that there is a significant difference between the values before and after the treatment in the one-class Trial. The broad class trial was carried out in class 5 as many as three categories, with 76 students. The Trial was carried out by conducting a pretest before doing online learning. Online learning was carried out using the Zenius 
application for one week; after learning, the Zenius application was carried out with a final test or posttest.

\begin{tabular}{|c|l|c|c|}
\hline NO & \multicolumn{1}{|c|}{ STUDENT NAME } & PRE TES & POST TEST \\
\hline 1 & Aditiya Bima Pratama & 40.00 & 78 \\
\hline 2 & Ahmad Annas Ashari & 48.00 & 79 \\
\hline 3 & Ahmad Fardhan Nitasal & 34.00 & 78 \\
\hline 4 & Amelia Dwi Rahma & 40.00 & 81 \\
\hline 5 & Dhafa Ananda Wahyu Ramadhan & 30.00 & 76 \\
\hline 6 & Dwi Ramadhani & 50.00 & 76 \\
\hline 7 & Eli Nirmala & 44.00 & 84 \\
\hline 8 & Ella Nafsiyah & 42.00 & 78 \\
\hline 9 & Ilham Harsena Ibrahim & 42.00 & 78 \\
\hline 10 & Islamie Bi Kafa & 34.00 & 78 \\
\hline 11 & Jofita Dwi Sariyah Ningsih & 34.00 & 78 \\
\hline 12 & Mohamad Athala Andriyan Muzakky & 36.00 & 78 \\
\hline 13 & Mohammad Hendra Setiawan & 38.00 & 82 \\
\hline 14 & Muhammad Imam Ghozali & 34.00 & 84 \\
\hline 15 & Muhammad Junior Putra Pratama & 28.00 & 86 \\
\hline 16 & Muhammad Rico Sugianto & 46.00 & 84 \\
\hline 17 & Muhammad Rosidil Muchlisin & 42.00 & 85 \\
\hline 18 & Nadia Salsabiil & 34.00 & 76 \\
\hline 19 & Putri Khoiriyah & 44.00 & 84 \\
\hline 20 & Revalina Marshanda & 52.00 & 85 \\
\hline 21 & Salsa Nabila Lestari Fajri & 40.00 & 85 \\
\hline 22 & Tanaya Eka Arum Sholichin & 38.00 & 81 \\
\hline 23 & Yoga Dwi Pratama & 38.00 & 87 \\
\hline 24 & Zahra Angel Arabella & 58.00 & 75 \\
\hline 25 & Zunita Dwi Ariyanti & 42.00 & 76 \\
\hline 1 & Mukhammad Afin Nurhidayat & 34.00 & 76 \\
\hline 2 & Ahmad Khusamuddin & 42.00 & 76 \\
\hline 3 & Avarel Muhamad Jamiludin & 36.00 & 76 \\
\hline 4 & Bangkit Taukhid Wahabain & & 79 \\
\hline & & & \\
\hline & & & \\
\hline
\end{tabular}




\begin{tabular}{|c|l|c|c|}
\hline 5 & Bima Ramadhani & 40.00 & 82 \\
\hline 6 & Devina Rahma & 42.00 & 76 \\
\hline 7 & Elok Fawzul Ishlahiyyah & 56.00 & 77 \\
\hline 8 & Fiana Dwi Lestari & 48.00 & 76 \\
\hline 9 & Frischa Arisha Putri & 44.00 & 95 \\
\hline 10 & Hariati Indriani & 28.00 & 78 \\
\hline 11 & Kquin Nara & 42.00 & 78 \\
\hline 12 & Manda Nurcahyani & 38.00 & 93 \\
\hline 13 & Mokhammad Fais & 38.00 & 78 \\
\hline 14 & Muhammad Arya & 50.00 & 77 \\
\hline 15 & Muhammad Fatkhur Roziqin & 50.00 & 80 \\
\hline 16 & Muhammad Ivan Putra Aditya & 46.00 & 78 \\
\hline 17 & Muhammad Johan Ramadani & 48.00 & 76 \\
\hline 18 & Nadia Indah Lestari & 42.00 & 97 \\
\hline 19 & Nur Adinda & 44.00 & 97 \\
\hline 20 & Rahma Ramadhani & 48.00 & 85 \\
\hline 21 & Rangga Adim Winata & 30.00 & 95 \\
\hline 22 & Reihan Divan Aditya & 44.00 & 93 \\
\hline 23 & Surya Farhandika Handoyo & 40.00 & 78 \\
\hline 24 & Teddy Vinc Sachviano & 40.00 & 75 \\
\hline 25 & Ufiyatul Fitria & 62.00 & 87 \\
\hline 1 & Achmad Adeleandro & 50.00 & 88 \\
\hline 2 & Ade Indri Fitriana & 32.00 & 82 \\
\hline 3 & Ahmad Diki Abdillah & 34.00 & 85 \\
\hline 4 & Angga Febri Saputra & 32.00 & 84 \\
\hline 5 & Azahra Riza Ramadina & 36.00 & 88 \\
\hline 6 & Bagus Muhammad Fatoni & 52.00 & 76 \\
\hline 7 & Defrian Sigit Widodo & 50.00 & 86 \\
\hline 8 & Dina Dwi Hidayah & 34.00 & 76 \\
\hline 9 & Elang Rigan Tandyo Ramadhan & 32.00 & 83 \\
\hline 10 & Fadel Arya Ghiyats & 54.00 & 89 \\
\hline 11 & Fatmareza Nadin Auliyanadita & 76 \\
\hline & & & \\
\hline
\end{tabular}




\begin{tabular}{|c|l|c|c|}
12 & Ferdiansyah Pramudya & 54.00 & 88 \\
\hline 13 & Gilang Sak Bana & 46.00 & 76 \\
\hline 14 & Intan Nur 'Aini & 52.00 & 77 \\
\hline 15 & Jerry Manggali Putra & 42.00 & 77 \\
\hline 16 & Khaliyah Ardana Putri & 56.00 & 87 \\
\hline 17 & Merri Tri Viana & 28.00 & 94 \\
\hline 18 & Mohammad Ilham Farizky & 60.00 & 81 \\
\hline 19 & Muhammad Ilham & 52.00 & 82 \\
\hline 20 & Muhammad Samsul Arifin & 44.00 & 76 \\
\hline 21 & Mukhammad Riki Ferdiansyah & 38.00 & 76 \\
\hline 22 & Nurlita Putri Dwi Ramadhanis & 44.00 & 76 \\
\hline 23 & Rahmat Wisnu Adi Pradana & 38.00 & 76 \\
\hline 24 & Tama Assa Maulana & 44.00 & 79 \\
\hline 25 & Vernanda Aprilian Hardiansyah & 36.00 & 78 \\
\hline 26 & Yunia Diana Ayu & 32.00 & 76 \\
\hline
\end{tabular}

\section{Descriptive Statistical Analysis}

\begin{tabular}{|c|c|c|c|c|}
\hline \multicolumn{5}{|c|}{ Descriptives } \\
\hline & & & Statistic & Std. Error \\
\hline \multirow[t]{13}{*}{ Nilai Pretest } & \multicolumn{2}{|l|}{ Mean } & 42.1316 & .90715 \\
\hline & \multirow{2}{*}{$\begin{array}{l}95 \% \text { Confidence Interval } \\
\text { for Mean }\end{array}$} & Lower Bound & 40.3244 & \\
\hline & & Upper Bound & 43.9387 & \\
\hline & \multicolumn{2}{|l|}{ 5\% Trimmed Mean } & 41.9474 & \\
\hline & \multicolumn{2}{|l|}{ Median } & 42.0000 & \\
\hline & \multicolumn{2}{|l|}{ Variance } & 62.542 & \\
\hline & \multicolumn{2}{|l|}{ Std. Deviation } & 7.90838 & \\
\hline & \multicolumn{2}{|l|}{ Minimum } & 28.00 & \\
\hline & \multicolumn{2}{|l|}{ Maximum } & 62.00 & \\
\hline & \multicolumn{2}{|l|}{ Range } & 34.00 & \\
\hline & \multicolumn{2}{|l|}{ Interquartile Range } & 12.00 & \\
\hline & \multicolumn{2}{|l|}{ Skewness } & .322 & .276 \\
\hline & \multicolumn{2}{|l|}{ Kurtosis } & -.371 & .545 \\
\hline \multirow[t]{13}{*}{ Nilai Posttest } & \multicolumn{2}{|l|}{ Mean } & 81.3421 & .67475 \\
\hline & \multirow{2}{*}{$\begin{array}{l}95 \% \text { Confidence Interval } \\
\text { for Mean }\end{array}$} & Lower Bound & 79.9979 & \\
\hline & & Upper Bound & 82.6863 & \\
\hline & \multicolumn{2}{|l|}{$5 \%$ Trimmed Mean } & 80.8509 & \\
\hline & \multicolumn{2}{|l|}{ Median } & 78.5000 & \\
\hline & \multicolumn{2}{|l|}{ Variance } & 34.601 & \\
\hline & \multicolumn{2}{|l|}{ Std. Deviation } & 5.88230 & \\
\hline & \multicolumn{2}{|l|}{ Minimum } & 75.00 & \\
\hline & \multicolumn{2}{|l|}{ Maximum } & 97.00 & \\
\hline & \multicolumn{2}{|l|}{ Range } & 22.00 & \\
\hline & \multicolumn{2}{|l|}{ Interquartile Range } & 9.00 & \\
\hline & \multicolumn{2}{|l|}{ Skewness } & 1.085 & .276 \\
\hline & \multicolumn{2}{|l|}{ Kurtosis } & .360 & .545 \\
\hline
\end{tabular}




\begin{tabular}{|c|c|c|c|}
\hline $\begin{array}{c}\text { Category } \\
\text { improvement }\end{array}$ & Category N-Gain & $\begin{array}{c}\text { Many } \\
\text { students }\end{array}$ & Percentage \\
\hline High & N-Gain $>0,7$ & 27 & $35.53 \%$ \\
\hline Low & $0,3=<$ N-Gain $=<0,7$ & 49 & $64.47 \%$ \\
\hline Medium & N-Gain $<0,3$ & 0 & $0.00 \%$ \\
\hline & & & $100.00 \%$ \\
\hline
\end{tabular}

\section{Prerequisite Test Data Normality}

From the pretest and posttest data, the normality of the data will be tested. Normality test or prerequisite test is used to test whether the variables are normally distributed or not to perform the t-test. If the data is not normally distributed, then the researcher must modify it first, but if it is usually distributed, then immediately perform the core test or t-test. The following is the calculation of the data normality test using SPSS.

\section{Tests of Normality}

\begin{tabular}{|l|r|r|r|r|r|c|}
\hline & \multicolumn{3}{|c|}{ Kolmogorov-Smirnov $^{\mathrm{a}}$} & \multicolumn{3}{c|}{ Shapiro-Wilk } \\
\cline { 2 - 7 } & Statistic & \multicolumn{1}{c|}{$\mathrm{df}$} & \multicolumn{1}{c|}{ Sig. } & \multicolumn{1}{c|}{ Statistic } & \multicolumn{1}{c|}{ df } & \multicolumn{1}{c|}{ Sig. } \\
\hline Nilai Pretest & .091 & 76 & .194 & .978 & 76 & .223 \\
Nilai Posttest & .215 & 76 & .000 & .853 & 76 & .000 \\
\hline
\end{tabular}

a. Lilliefors Significance Correction

Value of Sig. (P-Value) at the posttest value $<0.05$, it can be concluded that the posttest value is not normally distributed. So that the difference test does not use the t-test but the different test with nonparametric statistics.

\section{Uji nonparametric two related samples (Wilcoxon Signed Ranks Test)}

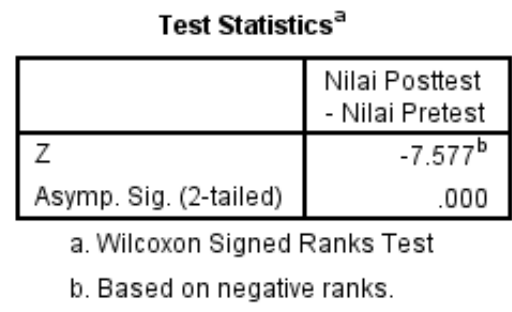

asymp. Sig. (2-tailed) $/ \mathrm{p}$-value $<0.05$, it can be concluded that there is a significant difference between the values before and after the treatment in the broad class trial. 


\section{Conclusion}

from the explanation above, it can be concluded that The results showed that from the limited Trial, the pretest and posttest scores increased with a high significance of $16.67 \%, 0 \%$, and low, $83.33 \%$. Then it can be said to be effective. In the usage trial, it is known that there is an increase in the pretest and posttest scores with a high significance level of $30 \%$, moderate $0 \%$, and low $70 \%$; it is stated that the use of the Zenius application in learning is effective. The broad class trial found an increase in the pretest and posttest scores with a high significance of $35.53 \%$, moderate $0 \%$, and low $64,47 \%$. it is stated that the application used in online learning is a practical.

\section{Reference}

[1] N. Mona, "Konsep Isolasi Dalam Jaringan Sosial Untuk Meminimalisasi Efek Contagious (Kasus Penyebaran Virus Corona di Indonesia)," J. Sos. Hum. Terap., vol. 2, no. 2, pp. 117-125, 2020.

[2] dkk Suwarno, "Implementation of Covid-19 on Character Education," J. Soc. Sci., vol. 2, no. 3, pp. 312-319, 2021.

[3] dkk Nur Harizah Zain, "Problematika Pembelajaran Daring pada Peserta Didik di Sekolah Dasar," J. Basicedu, vol. 5, no. 4, pp. 1840-1846, 2021.

[4] Afip Miftahul Basar, "Problematika Pembelajaran Jarak Jauh Pada Masa Pandemi Covid-19 (Studi Kasus di SMPIT Nurul Fajri - Cikarang Barat - Bekasi)," Edunesia J. Ilm. Pendidik., vol. 2, no. 1, pp. 208-218, 2021.

[5] dkk Moh. Irmawan Jauhari, "Problematika Pembelajaran Daring Di Madrasah Ibtidaiyah Negeri 3 Magetan," J. Educ. Relig. Stud., vol. 1, no. 1, pp. 9-15, 2021.

[6] dkk Rudi Haryadi, "PROBLEMATIKA PEMBELAJARAN DARING DI MASA PANDEMI COVID-19," AoEJ Acad. Educ. J., vol. 12, no. 2, pp. 254-261, 2021.

[7] Sugiyono, Metode Penelitian Kuantitatif, Kualitatif, $R \&$ D. Bandung: CV Alfabeta, 2017. 